\title{
Fluid-structure interaction analysis of gravity-based structure (GBS) offshore platform with partitioned coupling method. ${ }^{\star 2}$
}

\author{
W.Z. Lim ${ }^{\mathrm{a}, *}$, R.Y. Xiao ${ }^{\mathrm{b}}$ \\ ${ }^{a}$ Advanced Sustainable Manufacturing Technologies (ASTUTE), College of Engineering, \\ Swansea University, Swansea SA2 8PP, United Kingdom \\ ${ }^{b}$ School of The Built Environment and Architecture, London South Bank University, \\ Borough Road, London SE1 OAA, United Kingdom
}

\begin{abstract}
Fluid structure interaction (FSI) analysis is of great significance with the advance of computing technology and numerical algorithms in the last decade. This multidisciplinary problem has been expanded to engineering applications such as offshore structures, dam-reservoirs and other industrial applications. The motivation of this research is to investigate the fundamental physics involved in the complex interaction of fluid and structural domains by numerical simulations and to tackle the multiple surface interactions of a one-way coupling FSI GBS engineering case. To solve such problem, the partitioned method has been adopted and the approach is to utilise the advantage of the existing numerical algorithms in solving the complex fluid and structural interactions. The suitability has been validated for both strong and weak coupling methods which are the distinctive partitioned coupling approach. Therefore, with the computational platform of ANSYS FEA, the coupled field methods were adopted in this numerical analysis. Comparisons were made with the results obtained to justify the ability of both strong and weak methods in resolving the one-way coupling example with the potential applications in the field of ocean and marine engineering.
\end{abstract}

Keywords: fluid-structure interaction, offshore structure,partitioned

\footnotetext{
"Fully documented templates are available in the elsarticle package on CTAN.

*W.Z. Lim

Email address: w.z.lim@swansea.ac.uk ( W.Z. Lim)
} 
method

\section{Introduction}

Fluid structure interaction is a complex multi-physics phenomenon with contiguous domains consisting generally on fluid flow and solid structures with interaction between them. The structure deforms due to fluid action;

5 in the design of offshore structure engineering either from the research or industry applications such as offshore structures (Jo et al., 2013), wind turbine (Zhang et al., 2014) and ship structure (Ma and Mahfuz, 2012). The complexity of the offshore structures under a challenging environment of high seawater pressure impact has led to the development of robust numerical solvers for fluid-structure interaction problems. The numerical methods can be distinguished as either monolithic or partitioned method. In the monolithic approach, the interaction between the fluid and structure domain is treated synchronously under the interaction domain and are discretized in time and 15 space in the same manner. Information is exchanged on the interface synchronously and this single solution equation can be solved simultaneously for the fluid flow and displacement of the structure implicitly. This fully-coupled or direct approach is known to be highly robust and stable for a very strong fluid-structure interaction analysis such as in the research (Michler et al., 2004) and (Walhorn et al., 2005). However, monolithic method represents less modularity and require more coding than partitioned approach in which flow and structural equations are solved by using independent suitable algorithms and discretization methods. The partitioned method is an approach of which the two distinctive solvers (fluid and structure) are solved separately ture equations are integrated in time and the interface conditions are enforced asynchronously which means that the fluid flow does not change while the solution of the structural equations is calculated and vice versa. This approach preserves the software modularity and requires a coupling algorithm to allow for the interaction and to determine the solution of the coupled problem where information can be transferred between the two solvers. In general, this approach can be categorized into weakly or strongly coupled problem (Benra et al., 2011). There are two types of coupling systems in weak coupling such as the one-way and two-way coupling system. In the context of 
considered in the case of a single directional load which affects only from a particular field to another, for an example the fluid, $S_{f}$ and the structure, $S_{s}$ interaction of one-way coupling system as shown in Fig. 1 below (Richter, 2010).

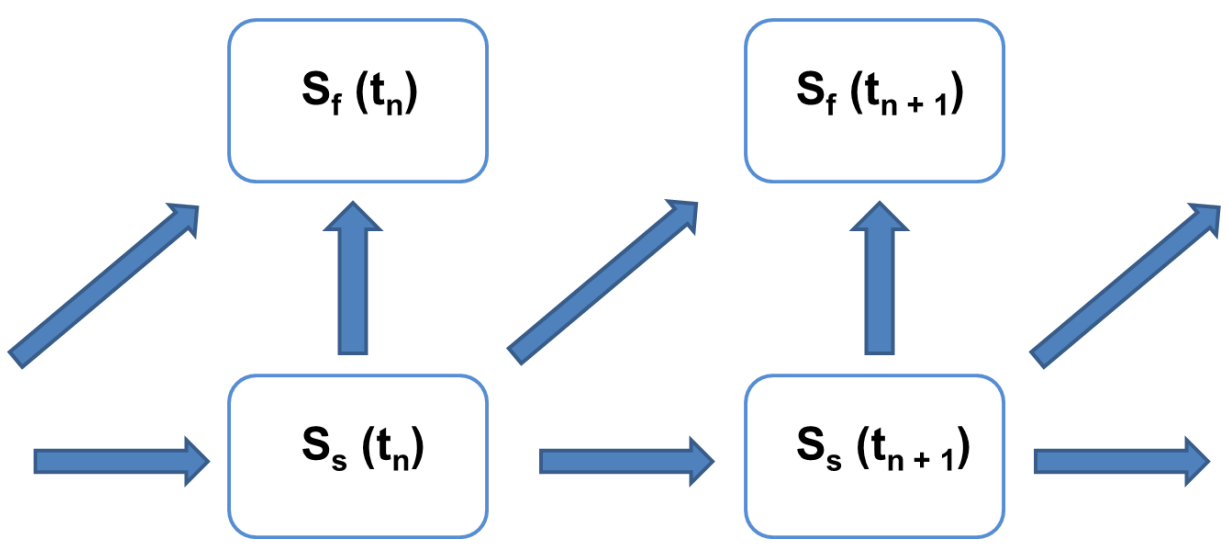

Fig. 1 Weakly coupled system for the one-way partitioned method

The interface between both domains is crucial and in most applications, a strong coupling system is approachable and this decoupling system allows parallel solution of the fluid and the structure domains. A strongly coupled system is a further development of the partitioned approach that two domains are solved independently in a decoupled way with an iterative interaction loop between each time-steps as indicated in Fig. 2. One advantage of the partitioned approach is that different solvers and schemes can be used for different multi-physics fields such as Habchi et al. (2013) Degroote et al. (2010)Song et al. (2013)Wall et al. (2007)Dettmer and Peri (2008).

\section{Mathematical model}

The governing equations of fluid and structural mechanics are presented in association with the Finite Element Method, Lagrangian Formulation and the Arbitrary Lagrangian-Eulerian formulation. These computational techniques are further implemented in solving the environment of the offshore FSI analysis solution within the framework of ANSYS APDL. 


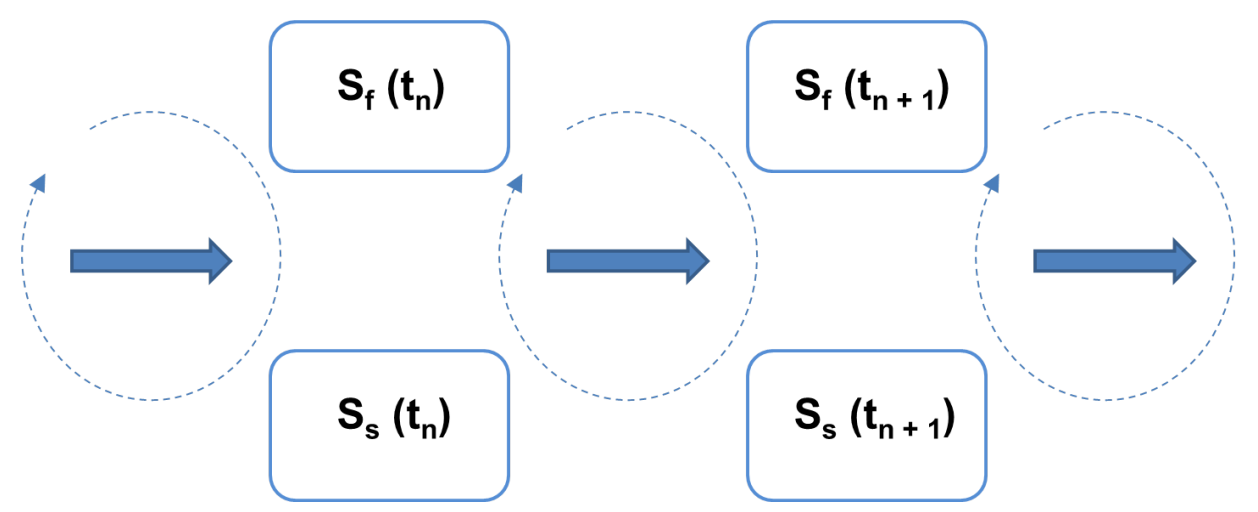

Fig. 2 Strongly coupled system for the one-way partitioned method

\subsection{Governing equations of fluid mechanics}

The fluid flow is defined by the laws of conservation of mass, momentum, and energy. Such laws are expressed in terms of partial differential equations which are discretised through finite element scheme ANSYS (2013). The fluid flow equations are governed by Navier-Stokes equations of incompressible flow.

\subsubsection{Continuity equation}

The continuity equation of the fluid flow is shown as:

$$
\frac{\partial \rho}{\partial t}+\nabla \cdot(\rho \boldsymbol{v})=0,
$$

where $\boldsymbol{v}$ is the velocity vectors for component in the $\mathrm{x}, \mathrm{y}$ and $\mathrm{z}$ directions. $\rho$ is the density of the fluid and $t$ is the time shown in the equation above. The rate of change of density can be replaced by the rate of change of pressure:

$$
\frac{\partial \rho}{\partial t}=\frac{\partial \rho}{\partial P} \frac{\partial P}{\partial t} .
$$

As for the incompressible solution:

$$
\frac{d \rho}{d P}=\frac{1}{\beta},
$$

where $P$ is the pressure and $\beta$ is bulk modulus of the fluid flow. 


\subsubsection{Momentum equation}

In a Newtonian fluid, the relationship between the stress and rate of deformation of the fluid is shown as:

$$
\boldsymbol{\tau}=[\lambda \operatorname{tr}(\nabla \boldsymbol{u})-P] \boldsymbol{I}+\mu\left(\nabla \boldsymbol{u}+\nabla \boldsymbol{u}^{\boldsymbol{T}}\right),
$$

where $\boldsymbol{\tau}, P, \boldsymbol{u}, \mu$ and $\lambda$ represent the stress tensor, the fluid pressure, orthogonal velocity vectors, dynamic viscosity and second coefficient of viscosity, respectively. The product of the second coefficient of viscosity and the divergence of the velocity is zero for a constant density fluid. Equation (4) transforms the momentum equations to the Navier-Stokes equations as follows:

$$
\frac{\partial \rho \boldsymbol{v}}{\partial t}+\nabla \cdot(\rho \boldsymbol{v} \otimes \boldsymbol{v})=\rho \boldsymbol{g}-\boldsymbol{P}+\boldsymbol{R}+\nabla \cdot\left(\mu_{e} \nabla \boldsymbol{v}\right)+\boldsymbol{T}
$$

where $\boldsymbol{g}, \boldsymbol{R}$ and $\boldsymbol{T}$ represent the acceleration vector due to gravity, distributed resistances and viscous loss terms vectors. The density of the fluid properties and effective viscosity are presented as $\rho$ and $\mu_{e}$ respectively. The viscous loss terms vector, $\boldsymbol{T}$ for all coordinate directions are eliminated in the incompressible, constant property case. The order of the differentiation is reversed in each term, reducing the term to a derivative of the continuity equation, which is zero.

\subsubsection{Turbulence}

Turbulence occurs when the inertial effects are significant enough with respect to viscous effects and the instantaneous velocity being fluctuate at every point in the flow field. The velocity is thus expressed in terms of a mean value and a fluctuating component:

$$
V_{x}=\bar{V}_{x}+V_{x}^{\prime}
$$

where $\bar{V}_{x}$ and $V_{x}^{\prime}$ are the mean component of velocity and fluctuating component of velocity in $\mathrm{x}$-direction respectively. In the Navier-Stokes equations, the instantaneous velocity equation is time averaged where the fluctuating component is zero and the time average of the instantaneous value is the average value. The time interval for the integration is arbitrarily chosen as shown below:

$$
\frac{1}{\Delta t} \int_{0}^{\delta_{t}} V_{x}^{\prime} d t=0 ; \frac{1}{\delta_{t}} \int_{0}^{\delta_{t}} V_{x} d t=\bar{V}_{x} .
$$


After the substitution of Equation (6) into the momentum equations, the time averaging leads to additional terms and the extra terms are:

$$
\boldsymbol{\sigma}^{\boldsymbol{R}}=-\nabla \cdot\left(\rho \boldsymbol{V}^{\prime} \otimes \boldsymbol{V}^{\prime}\right)
$$

where $\boldsymbol{\sigma}^{\boldsymbol{R}}$ is the Reynolds stress terms. The standard k-model is applied where the turbulent viscosity, $\mu_{t}$ is calculated as a function of the turbulence parameters kinetic energy $k$ and its dissipation rate using the Equation (9) below where $C_{\mu}$ is the turbulence constant and $\epsilon$ is the turbulent kinetic energy dissipation rate.

$$
\mu_{t}=C_{\mu} \rho \frac{k^{2}}{\epsilon}
$$

\subsubsection{Pressure}

For the calculation of the pressure, the defining expression for the relative pressure is:

$$
P_{a b s}=P_{r e f}+P_{r e l}-\rho_{o} \cdot \boldsymbol{g} \cdot \boldsymbol{r}+\frac{1}{2} \rho_{o}(\boldsymbol{\omega} \times \boldsymbol{\omega} \times \boldsymbol{r}) \cdot \boldsymbol{r} .
$$

Combining the momentum equations into vector form, the result is obtained as:

$$
\rho \frac{D \boldsymbol{\nu}}{D t}+2 \rho \boldsymbol{\omega} \times \boldsymbol{\nu}+\rho \boldsymbol{\omega} \times \boldsymbol{\omega} \times \boldsymbol{r}=\rho \boldsymbol{g}-\nabla P_{a b s}+\mu \boldsymbol{\nabla} \cdot(\boldsymbol{\nabla} \boldsymbol{\nu}),
$$

where $\rho_{o}, P_{r e f}, \boldsymbol{g}, P_{a b s}, P_{r e l}, \boldsymbol{r}, \boldsymbol{w}, \boldsymbol{v}, \mu$, and $\boldsymbol{\rho}$ are the reference density, reference pressure, gravity vector, absolute pressure, relative pressure, position vector of fluid particle relating to rotating coordinate system, angular velocity vector, velocity vector in global coordinate system, fluid viscosity and fluid density respectively. For the case of coupling in fluid flow, moving interfaces are included with the effect on the structural deformation which will deform the fluid mesh. Such phenomenon changes with time and needs to satisfy the boundary conditions at the moving interfaces, Arbitrary LagrangianEulerian (ALE) formulation Donea and Huerta (2003) is applied in solving such problems and the examples can be found in Bathe and Zhang (2009).

\subsubsection{Arbitrary Lagrangia-Eulerian, ALE algorithms}

The Eulerian algorithms are widely used in fluid mechanics where the computational mesh is fixed and the fluid moves along with the grid. Unlike the Lagrangian algorithms, these algorithms facilitate the treatment of 
large distortions in the fluid motion and are indispensable for the simulation of turbulent flows. On the contrary, it has the difficulty to follow free surface(s) and interface(s) between different materials or different media for example the fluid to fluid and fluid to solid interfaces. Therefore, we have ruled out such algorithms rather than only considering the ideal Arbitrary Lagrangian-Eulerian algorithms as the key solution of fluid domain and the fluid-structure interfaces instead Donea and Huerta (2003). Fluid flow problems often involve moving interfaces which include moving internal walls (for example, a solid moving through a fluid), external walls or free surfaces. ALE formulations are used to solve the problems where the fluid domain of the seawater changes with time and movement of finite element to satisfy the boundary conditions at the moving interface(s). The Eulerian equations of motion need to be modified to reflect the moving frame of reference. The time derivative terms are essentially rewritten in terms of the moving frame of reference where $\phi$ and $\boldsymbol{W}$ are the degree of freedom and velocity of the moving frame of reference, respectively as shown below:

$$
\left.\frac{\delta \phi}{\delta t}\right|_{\text {fixed frame }}=\left.\frac{\delta \phi}{\delta t}\right|_{\text {moving frame }}-\boldsymbol{W} \cdot \boldsymbol{\nabla} \phi .
$$

\subsubsection{Segregation solution algorithm}

For coupling algorithm, the pressure and momentum equations are coupled with the SIMPLEF algorithm originally belong to a general class referred to as the Semi-Implicit Method for Pressure Linked Equations (SIMPLE), see Versteeg and Malalasekera (2007). The incompressible algorithm is a special case of the compressible algorithm. The change in the product of density and velocity from each iteration is approximated by the considering changes separately through a linearization process as shown in ANSYS (2013):

\subsection{Governing equations of structural mechanics}

The offshore structure equation is based on the impulse conservation that is solved by using a finite element approach as shown below where $\boldsymbol{M}, \boldsymbol{C}, \boldsymbol{K}$, $\ddot{\boldsymbol{u}}, \dot{\boldsymbol{u}}$ and $\boldsymbol{u}$ are the mass, damping coefficient, stiffness, acceleration, velocity, and displacement vectors, respectively:

$$
\boldsymbol{M} \cdot \ddot{\boldsymbol{u}}+\boldsymbol{C} \cdot \dot{\boldsymbol{u}}+\boldsymbol{K} \cdot \boldsymbol{u}=\boldsymbol{F} .
$$

\subsection{1. von Mises yield criterion}

The von Mises yield criterion known as the octahedral shearing stress is a convenient alternative choice to the maximum shearing stress as the key 
variable for causing yield of materials which are pressure-independent. The von Mises or equivalent strain $\varepsilon_{e}$ is computed as:

$$
\varepsilon_{e}=\frac{1}{1+\nu^{\prime}}\left(\frac{1}{2}\left[\left(\varepsilon_{1}-\varepsilon_{2}\right)^{2}+\left(\varepsilon_{2}-\varepsilon_{3}\right)^{2}+\left(\varepsilon_{3}-\varepsilon_{1}\right)^{2}\right]\right)^{\frac{1}{2}}
$$

where $\varepsilon_{1}, \varepsilon_{2}$ and $\varepsilon_{3}$ are the three principal strains and $\nu^{\prime}$ is the effective Poisson's ratio. The equivalent stress (von Mises) related to the principal stress can be obtained from

$$
\sigma_{e}=\left(\frac{1}{2}\left[\left(\sigma_{1}-\sigma_{2}\right)^{2}+\left(\sigma_{2}-\sigma_{3}\right)^{2}+\left(\sigma_{3}-\sigma_{1}\right)^{2}\right]\right)^{\frac{1}{2}}
$$

or

$$
\sigma_{e}=\left(\frac{1}{2}\left[\left(\sigma_{x}-\sigma_{y}\right)^{2}+\left(\sigma_{y}-\sigma_{z}\right)^{2}+\left(\sigma_{z}-\sigma_{x}\right)^{2}+6\left(\sigma_{x y}^{2}+\sigma_{y z}^{2}+\sigma_{x z}^{2}\right)\right]\right)^{\frac{1}{2}}
$$

where $\sigma_{e}$ is the equivalent stress of any arbitrary three-dimensional stress state to be represented as a single positive stress values. The equivalent stress is part of the maximum equivalent stress failure theory known as yield functions which can be referred to Chen (2007). When $\nu^{\prime}=\nu$ the equivalent stress is related to the equivalent strain through

$$
\sigma_{e}=E \varepsilon_{e}
$$

\subsection{Coupling equations}

The interaction of the fluid seawater and the offshore structure at a mesh interface causes the pressure to exert a force applied to the structure and the structure motions produce an effective fluid load. The governing finite element matrix equations then become:

$$
\begin{array}{r}
{\left[\boldsymbol{M}_{\boldsymbol{s}}\right] \ddot{\boldsymbol{U}}+\left[\boldsymbol{K}_{\boldsymbol{s}}\right] \ddot{\boldsymbol{U}}=\boldsymbol{F}_{\boldsymbol{s}}+[\boldsymbol{R}] \boldsymbol{P}} \\
{\left[\boldsymbol{M}_{\boldsymbol{f}}\right] \ddot{\boldsymbol{P}}+\left[\boldsymbol{K}_{\boldsymbol{f}}\right] \ddot{\boldsymbol{P}}=\boldsymbol{F}_{f}-\rho_{o}[\boldsymbol{R}]^{T} \ddot{\boldsymbol{U}}}
\end{array}
$$

where $\boldsymbol{M}, \ddot{\boldsymbol{U}}, \boldsymbol{K}, \boldsymbol{F}$ and $\boldsymbol{P}$ are the mass, acceleration, stiffness, force and pressure respectively. $[\boldsymbol{R}]$ is a coupling matrix that represents the effective surface area associated with each node on the fluid-structure interface (FSI). 
The coupling matrix $[\boldsymbol{R}]$ also takes into account the direction of the normal vector defined for each pair of coincident fluid and structural element faces that comprises the interface surface. The positive direction of the normal vector, as the program uses it, is defined to be outward from the fluid mesh and inwards to the structure. Both the structural and fluid load quantities that are produced at the fluid-structure interface are functions of unknown nodal degrees of freedom. Placing these unknown load quantities on the left hand side of the equations and combining the two equations into a single equation produces the following:

$$
\left[\begin{array}{cc}
\boldsymbol{M}_{\boldsymbol{s}} & 0 \\
\rho_{o} \boldsymbol{R}^{\boldsymbol{T}} & \boldsymbol{M}_{\boldsymbol{f}}
\end{array}\right]\left(\begin{array}{c}
\ddot{\boldsymbol{U}} \\
\ddot{\boldsymbol{P}}
\end{array}\right)+\left[\begin{array}{cc}
\boldsymbol{K}_{\boldsymbol{s}} & -\boldsymbol{R} \\
0 & \boldsymbol{K}_{\boldsymbol{f}}
\end{array}\right]\left(\begin{array}{l}
\boldsymbol{U} \\
\boldsymbol{P}
\end{array}\right)=\left(\begin{array}{c}
\boldsymbol{F}_{\boldsymbol{s}} \\
\boldsymbol{F}_{\boldsymbol{f}}
\end{array}\right) .
$$

The foregoing equation implies that nodes on a fluid-structure interface have both displacement and pressure degrees of freedom.

\section{Gravity based offshore structure, GBS platform}

The offshore gravity-based structure (GBS) platform was modelled which was based on the MARINTEK, Norway three dimensional model Stansberg et al. (2005) Baarholm and Stansberg (2004). The aim of this three dimensional numerical analysis was to understand the behaviour of the offshore structure and to further validate the suitability of both strong and weak partitioned coupling methods and to make comparison that involved multiple interaction surfaces. The GBS platform was coupled with the attempt of both strong and weak coupling techniques undergo high wave impact from the recent Tohoku, Japan, 2011 earthquake that induced a high velocity inflow. The actual recorded peak acceleration of $26.49 \mathrm{~m} / \mathrm{s}^{2}$ at $t=1.10 \mathrm{~s}$ Kalkan and Sevilgen (2011) was taken having the peak velocity of $29.99 \mathrm{~m} / \mathrm{s}$. The hypothetical model of the GBS platform can best be referred to Fig. 3 and the material properties of the offshore structure GBS platform was assumed as a high strength concrete with the properties stated in Table 1 as shown.

This one-way coupling example of FSI with the strong and weak partitioned techniques concentrated on the interactions between the solid domain, GBS platform surface(s) with the fluid domain of seawater that inducing the high velocity fluid flow. The movement and deformation of the structure

is trivial in this case. The results from numerical analyses of the strong 


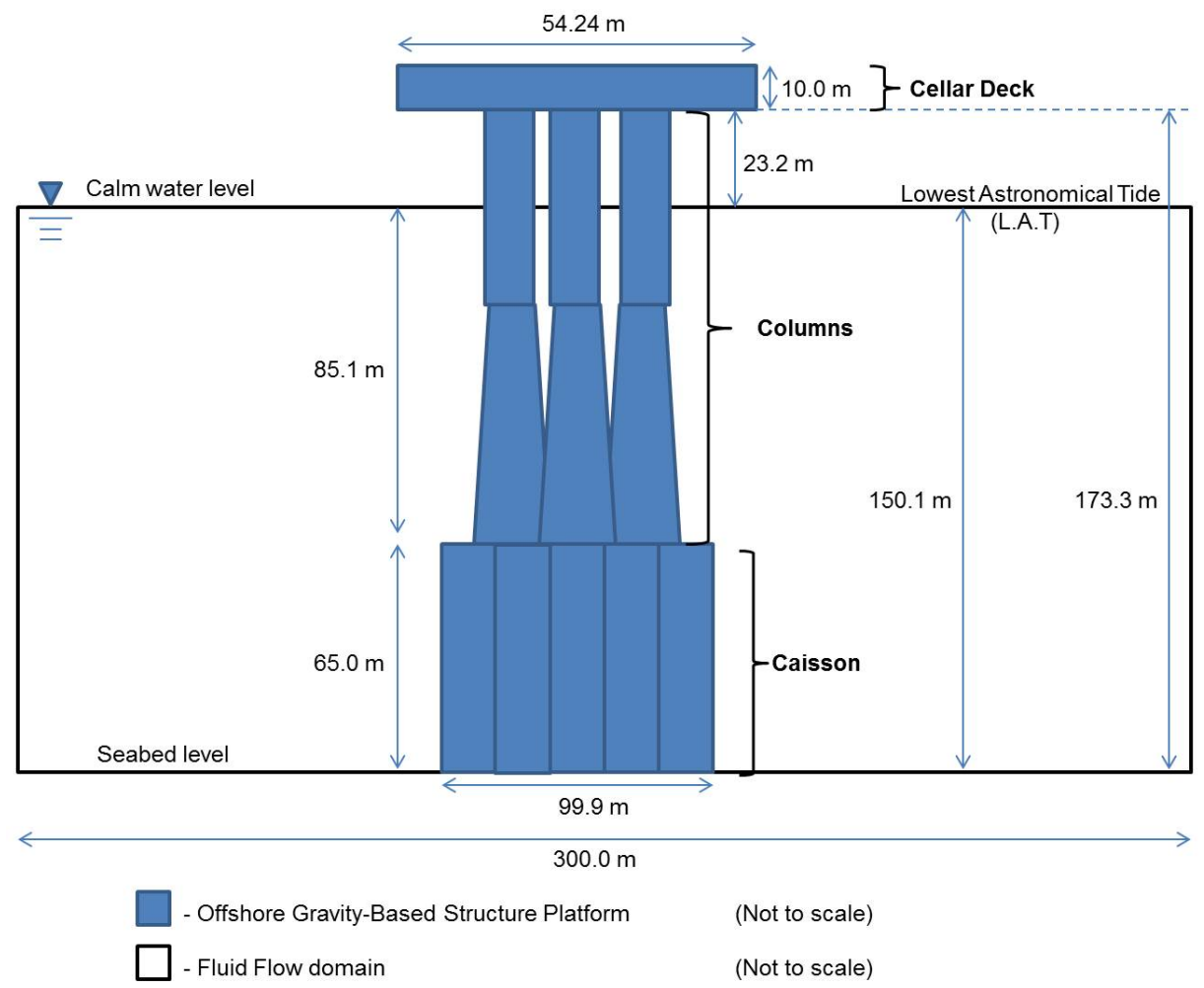

Fig. 3 Hypothetical model of the offshore gravity-based structure (GBS) platform (the Statfjord A GBS platform, North Sea) from MARINTEK, Norway and the sea-water level.

and weak coupling system will be compared in respective to hydrodynamics pressure and von Mises stress obtained in the later section.

\section{Numerical model}

In the computational application of the GBS offshore structure, ANSYS APDL was chosen to be the computing platform where the corresponding element types that were used are element SOLID185 for the offshore GBS platform and element FLUID142 for the sea water under high wave impact fluid flow domain. The SOLID185 element is defined as eight nodes having three degrees of freedom at each node: translations in the nodal $\mathrm{x}, \mathrm{y}$, and $\mathrm{z}$ directions. Whereas the FLUID142 element is defined by eight nodes with the degree of freedom; velocities and pressure. Both SOLID185 and 
Table. 1 Materials properties for the gravity-based offshore structure, GBS

\begin{tabular}{|c|c|c|c|c|}
\hline Material & $\begin{array}{c}\text { Elastic Modulus } \\
(\mathbf{M P a})\end{array}$ & Poisson Ratio's & $\begin{array}{c}\text { Density } \\
\left(\mathrm{kg} / \mathrm{m}^{3}\right)\end{array}$ & $\begin{array}{c}\text { Viscosity } \\
(\mathrm{Pa.s})\end{array}$ \\
\hline Concrete (High-Strength) & 30 & 0.2 & 2400 & - \\
\hline Water & - & - & 1000 & 8.9 \\
\hline
\end{tabular}

FLUID142 elements are compatible in relation to the coupled-field method of fluid interacting with the solid structure in three dimensional forms.

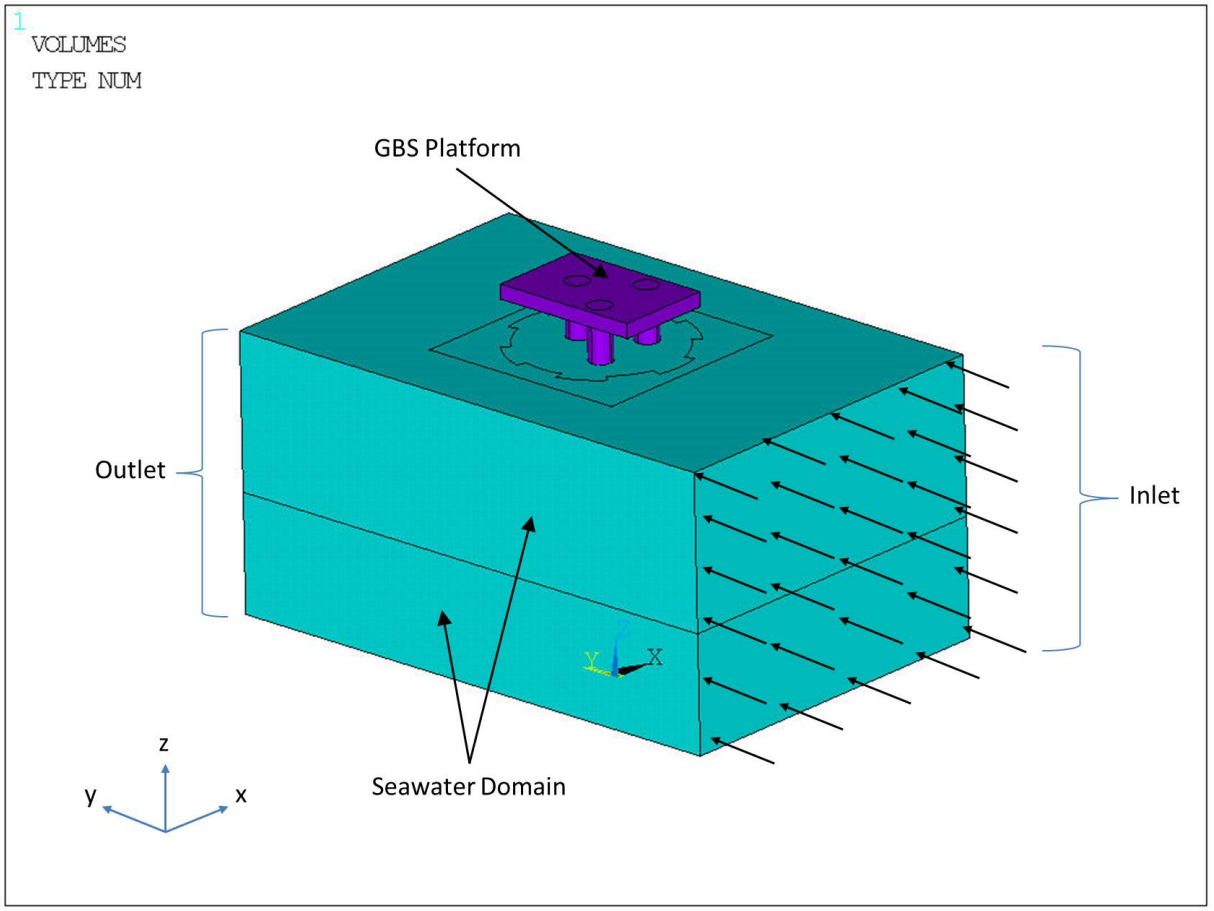

Fig. 4 Numerical geometry model of the GBS platform and sea-water domains. 


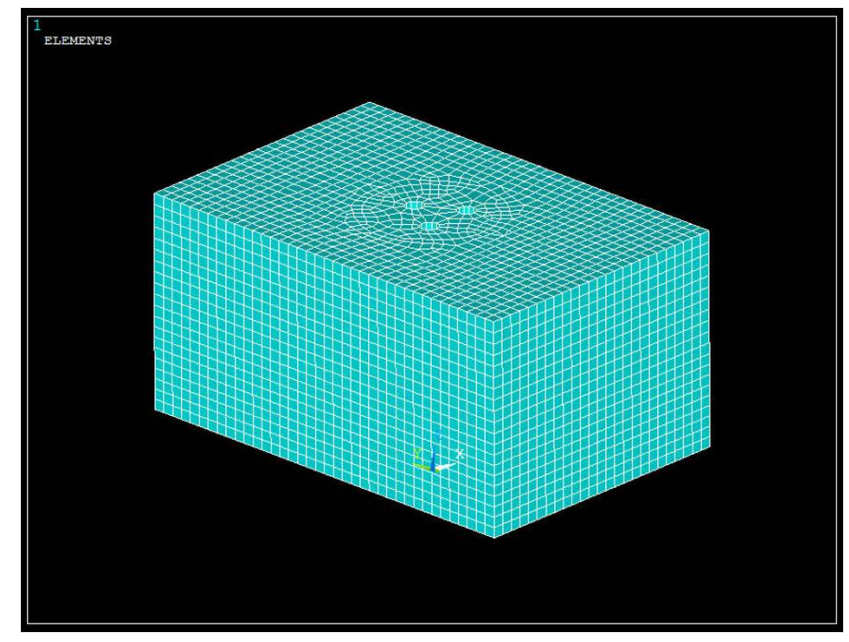

Fig. 5 Three-dimensional numerical model of the sea-water domain (FLUID142 elements).

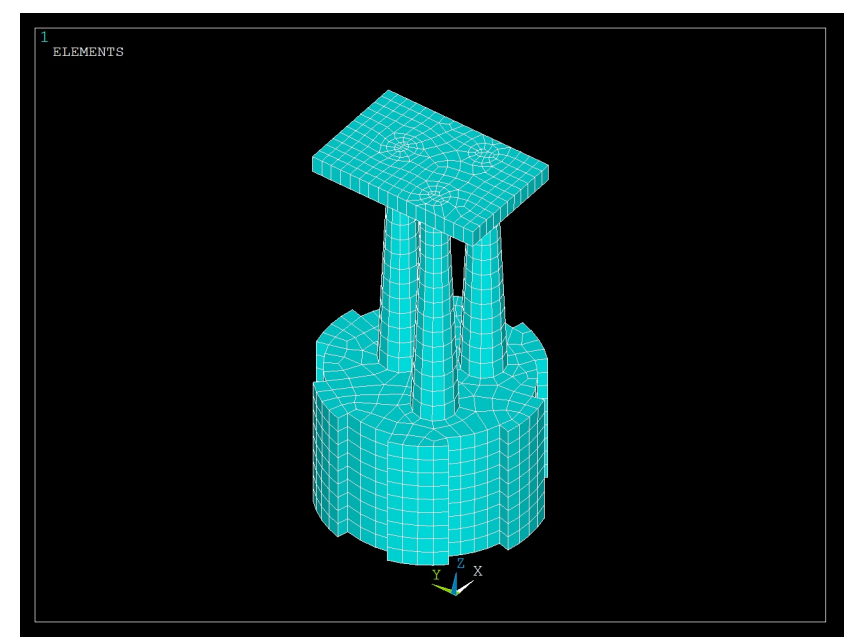

Fig. 6 Three-dimensional numerical model of the offshore GBS platform domain (SOLID185 elements). 
The geometry of the numerical model of the GBS platform and seawater is depicted in Fig. 4. The inlet velocity vector was assigned at the y-direction in the global coordinate system. Three dimensional SOLID185 elements provide the degree of freedom of deformation and von Mises stress in the discipline of structure analysis solver and FLUID142 elements provide the degree of freedom of velocity and hydrodynamics pressure in the discipline of FLOTRAN-CFD analysis solver. The element model and offshore GBS platform domain are illustrated in Fig. 5 and Fig. 6 respectively.

\subsection{Coupling methods}

The partitioned approach of coupling methods Multi-Field Single-Code, MFS and Load Transfer Physics Environment adapted in the ANSYS APDL platform are considered and compared for this numerical FSI problem. Such coupling methods had also been adopted by Lim et al. (2012) and Lim et al. (2013) for the problem of concrete gravity dam. The MFS coupling solver is specified as strong coupled system of partitioned method and the solution method is specifically shown in Fig. 7. It can be defined as a sequential coupled field system that is able to solve a lot of coupling analysis problems. The MFS solver was defined with twenty time-steps and within each time loop there is a stagger loop. The staggering loop allow for implicit coupling of the seawater and offshore structure fields in the MFS solution. The maximum twenty iterations of stagger iteration specified are to determine the convergence of the load transfer between fields. The displacement of the structure was defined as the convergence criteria for both strong and weak coupling analysis where the total displacement of the structure for each time-step must be greater or equal to zero. 


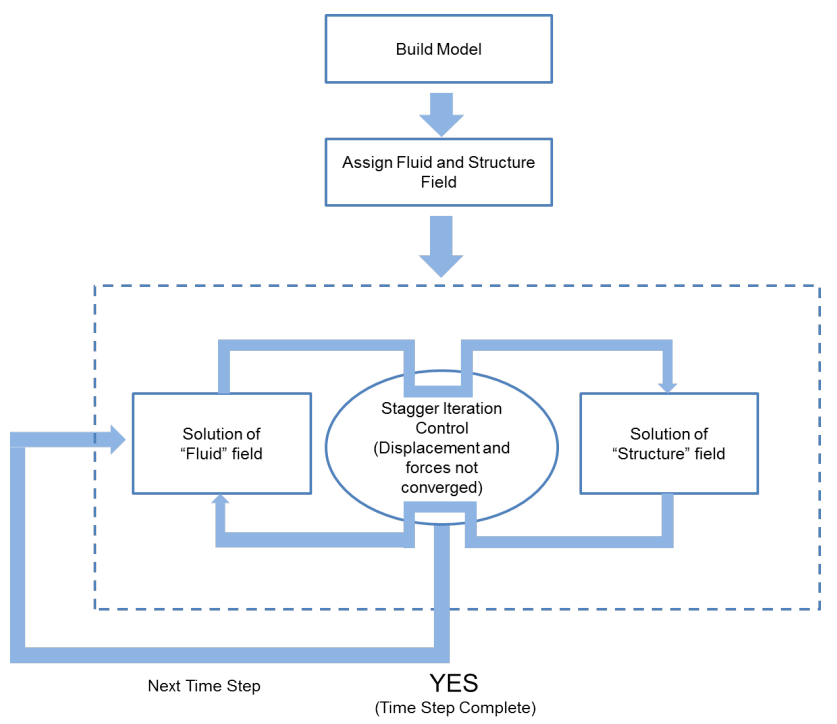

Fig. 7 Solution procedure of Multi-Field Single-Code coupling (MFS) for the FSI GBS offshore problem (Strong Coupling System).

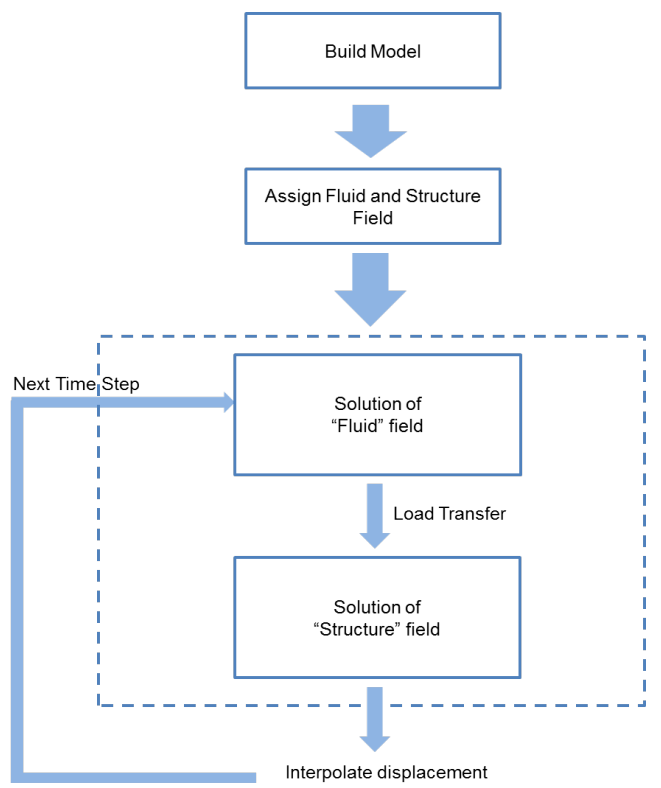

Fig. 8 Solution procedure of Load Transfer Physics Environment for the FSI GBS offshore problem (Weak Coupling System). 


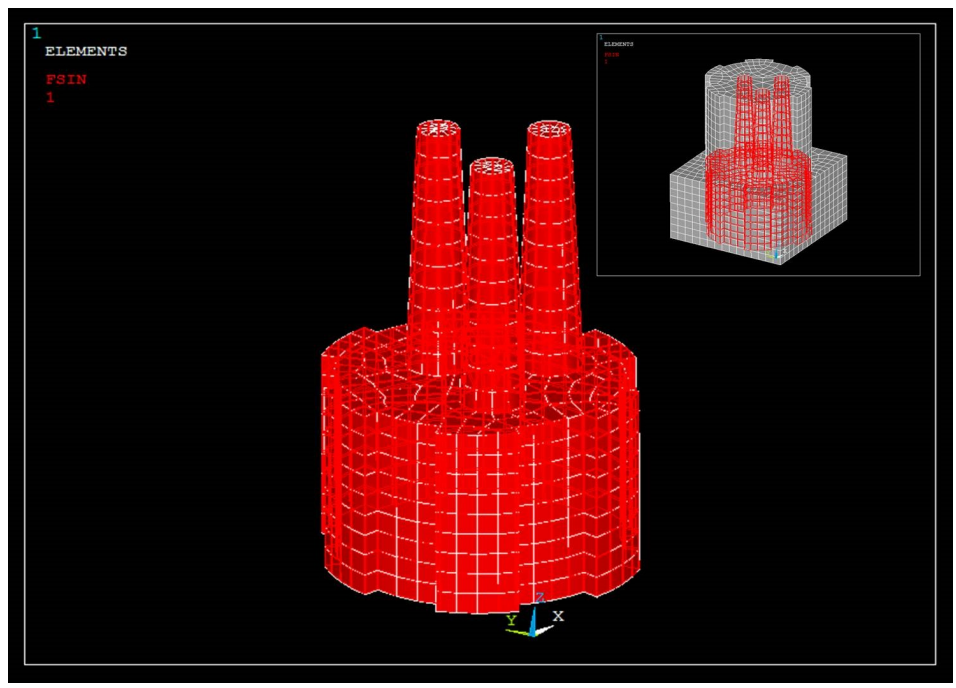

Fig. 9 Surface(s) interaction between the GBS surface(s) and seawater surface(s) where transformation of load vector force and displacement occur. 
The Load Transfer Physics Environment is consider as weak coupled system of partitioned method under a developed user looping system with the ANSYS parameter design language (APDL). The looping of the weak coupling system is shown in Fig. 8 and the input of one physics analysis depends on the results from another analysis. For the weak coupling system of Load Transfer Physics Environment, there is no staggering iteration between each time loop hence the solutions are easily converged within the maximum twenty time-steps specified. In this paper, the numerical problem is treated as a one-way coupling approach and both methods involve the multiple surface interactions between the fluid and structural domain especially at the columns and base surfaces shown in Fig. 9 .

\section{Results and discussion}

Based on the results of both coupled-field analyses, the distribution patterns of the hydrodynamics pressure and von Mises stress are fairly rational and agreeable for the offshore GBS numerical problem in terms of comparison. Such results was proven and shown in Fig. 10 for the hydrodynamics pressure of the sea water domain around the surface of the GBS offshore platform base as well as the build-up von Mises stresses on the overall GBS Offshore structure as shown in Fig. 11 for the strong and weak coupling approach.

Five different node locations were selected on the morphing region (seawater domain) and the offshore GBS structure domain as shown in Fig. 12 with the purpose of further verification and comparison of the both methods. Comparison results of the average hydrodynamics pressure and von Mises stress values for the different node locations are shown in Fig. 13 and Fig. 14. It clearly show that the distribution patterns for the pressures and stresses of both methods were rationally similar and close in comparison for all the node locations. Such indicate that the both coupling system are capable of solving high density of surface(s) interaction. In terms of value, the maximum average ratio for hydrodynamics pressure is 1.412 (Location Case 2 ) and for the von Mises stress is 1.670 (Location Case 4). The comparison results can best refer to Table 2 for better study on the overall average ratio of hydrodynamics pressure and the von Mises stress results as well. The overall average ratio for the hydrodynamics pressure and the von Mises stress are 1.260 and 1.280 respectively. 
Table. 2 Average ratio of hydrodynamics pressure and von Mises stress in comparison of both MFS and Load Transfer Physics Environment methods for the offshore GBS platform

\begin{tabular}{|c|c|c|}
\hline Location Case & Hydrodynamics Pressure & von Mises Stress \\
\hline 1 & 1.260 & 1.070 \\
\hline 2 & 1.412 & 1.190 \\
\hline 3 & 1.350 & 1.130 \\
\hline 4 & 1.228 & 1.670 \\
\hline 5 & 1.049 & 1.340 \\
\hline Overall average ratio & $\mathbf{1 . 2 6 0}$ & $\mathbf{1 . 2 8 0}$ \\
\hline
\end{tabular}

There were only a slight variations in the overall average ratio value between the comparison of both coupling methods. Hence, regardless of the increment of the surface interactions between the structure and fluid domain, the value obtained for both hydrodynamic pressure and von Mises stress for the weak and strong coupling are fairly similar. Somehow, the trends of both graphs oscillate with almost similar patterns and the distributions of 


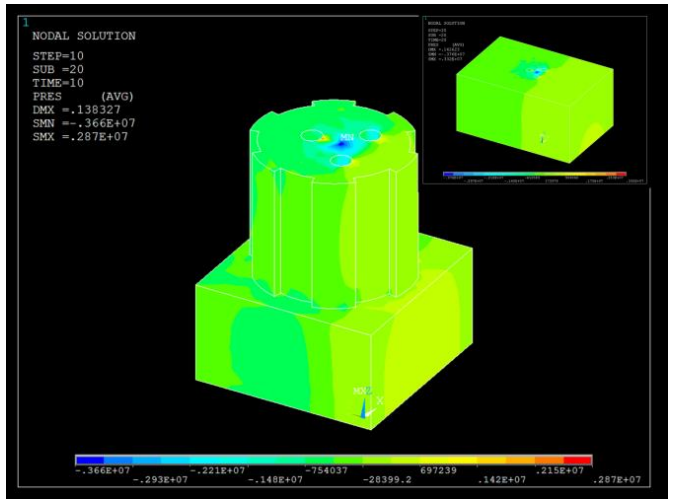

(a)

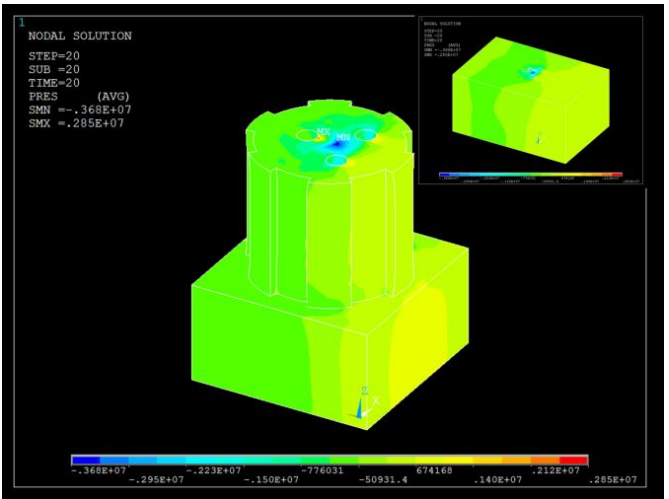

(b)

Fig. 10 Comparison of hydrodynamics pressure distribution results between (a) Multi-Field Single Code, MFS (Strong Coupling) and ; (b) Load Transfer Physics Environment (Weak Coupling) around the interaction surfaces at the GBS offshore platform base.

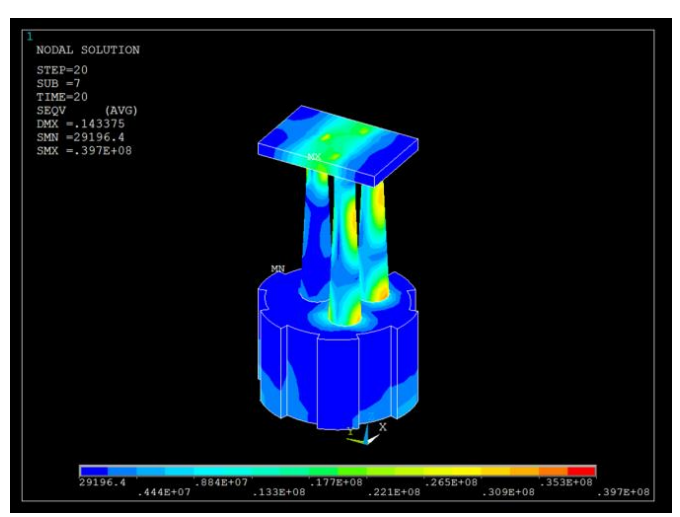

(a)

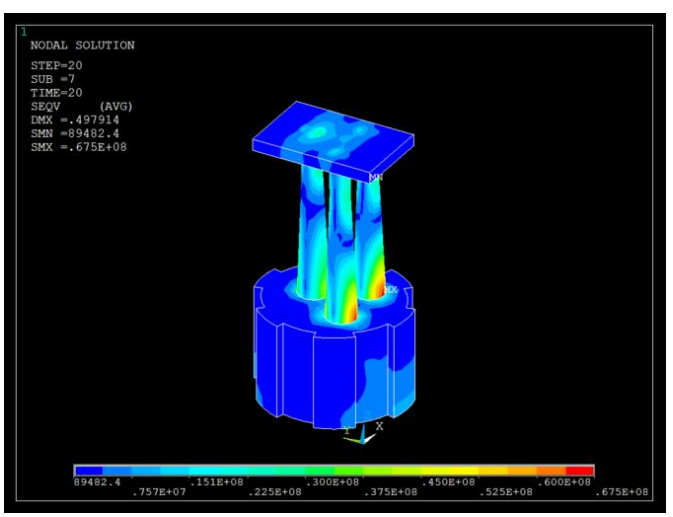

(b)

Fig. 11 Comparison of stress distribution results between (a) Multi-Field Single Code, MFS (Strong Coupling) and ; (b) Load Transfer Physics Environment (Weak Coupling) for the overall GBS offshore structure. 


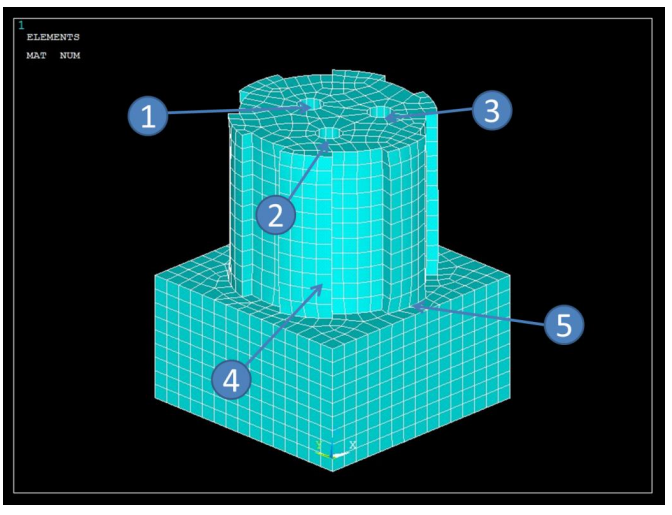

(a)

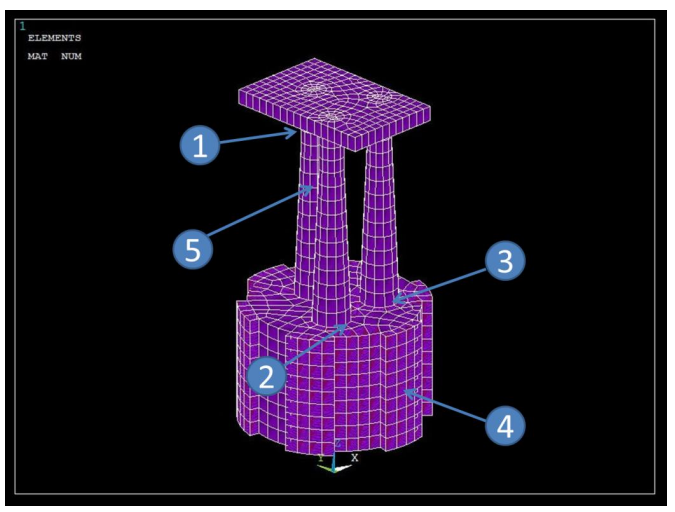

(b)

Fig. 12 Selected node locations of the (a) seawater (morphing region) domain on the hydrodynamics pressure results; (b) Offshore GBS platform on the von Mises stress results.

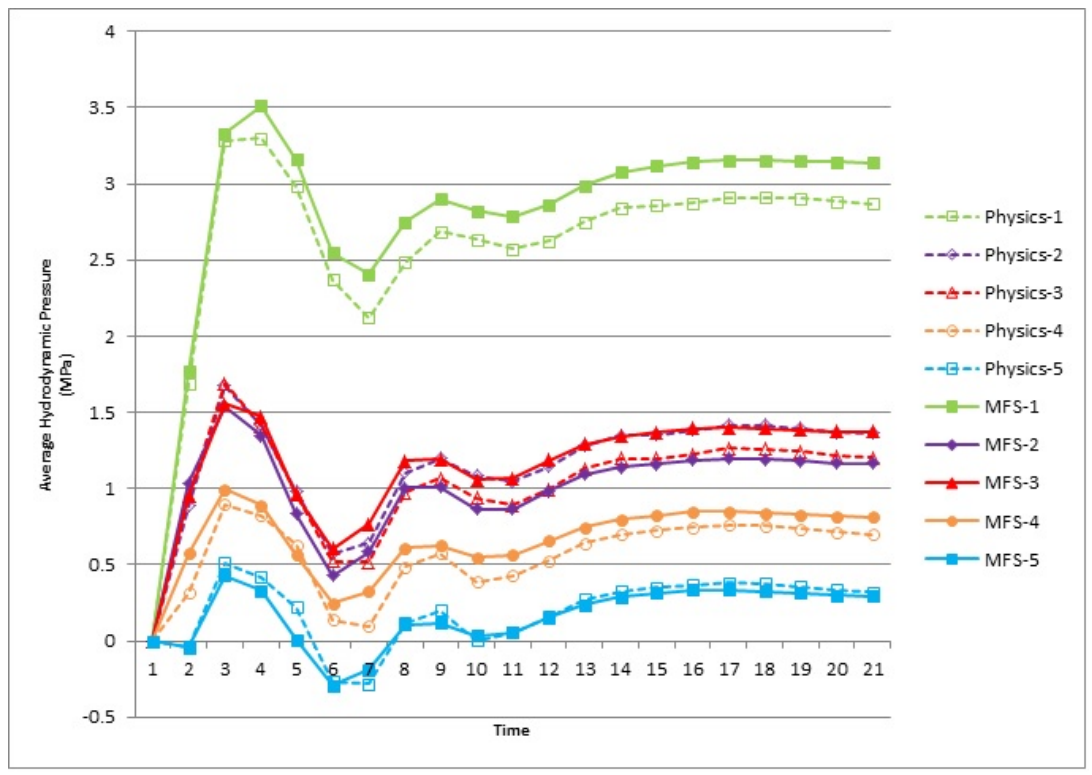

Fig. 13 Selected node locations of the (a) seawater (morphing region) domain on the hydrodynamics pressure results; (b) Offshore GBS platform on the von Mises stress results. 


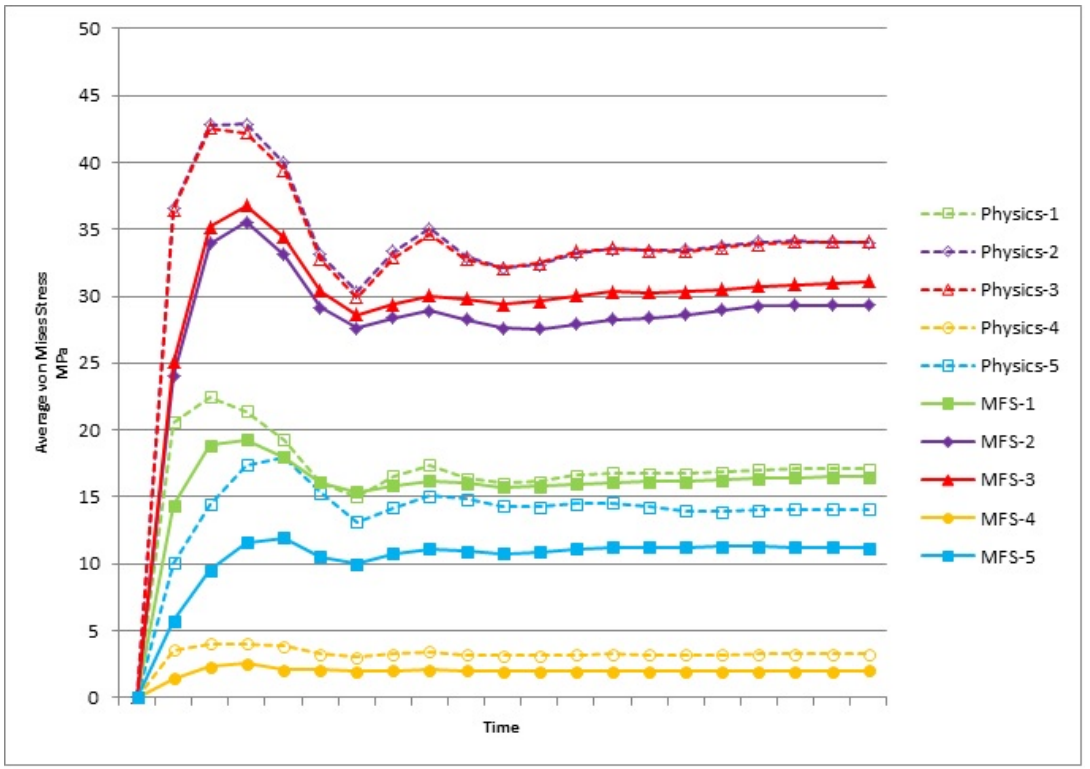

Fig. 14 Selected node locations of the (a) seawater (morphing region) domain on the hydrodynamics pressure results; (b) Offshore GBS platform on the von Mises stress results. 
high stresses are of the same occurring at the bottom and sides of the rigid concrete gravity-dam structure. Such results and analysis conclude that the overall one-way coupling could be resolved with the approach of both partitioned weak and strong coupling systems in the MFS and Load Transfer Physics Environment respectively. These proved that the weak and strong coupling methods are capable in solving the many problem of a oneway coupling offshore structure issue in carrying out feasibility design analysis especially under harsh pressure conditions .

\section{Conclusions}

The analysis of GBS platform used in this paper was to illustrate the differences between the coupling algorithms for both strongly and weakly coupled user developed system of the partitioned methods. These will enable feasible applications in the area of offshore and marine engineering structures. The scope and capability of both methods have been tested and compared from the numerical results obtained, it has proved that both weak and strong coupled field methods are oscillating with the same pressure and stress distributions that justify their capabilities of transferring load between the surfaces of interaction. In both examples, the offshore structures have responded to the pressure impact through the interaction surface or region with the distribution patterns being similar. However, the small differences of average ratio in the stress value could be caused by the stringent convergence in the strong coupling algorithm due to the multiple surface interactions of the numerical model whereas the weak coupling algorithm has loose convergence within the surface of interaction. The developed techniques of the weak coupling system has been proved to be more flexible in terms of the existing APDL in ANSYS which allow the development of parameters and algorithms proposed in this paper in tackling the one-way coupling FSI GBS Offshore platform problem. Hence, the user assigned one-way weak coupling algorithm can be an ideal method in solving both single and multiple scale of surface interactions in a feasible design analysis of an offshore or marine engineering field.

\section{Acknowledgements}

The authors would like to acknowledge the support of London South Bank University in allowing this research to be conducted. The first author would also like to acknowledge the scholarship provided by the London South Bank University and the support of Swansea University. 
ANSYS (2013). Documentation Release 14.5.

Baarholm, R. and Stansberg, C. T. (2004). Extreme vertical wave impact on the deck of a gravity-based structure (GBS) platform. Rogue Waves.

Bathe, K. J. and Zhang, H. (2009). A mesh adaptivity procedure for CFD 240 and fluid-structure interactions. Computers \& Structures, 87(1112):604 617.

Benra, F. K., Dohmen, H. J., Pei, J., Schuster, S., and Wan, B. (2011). A comparison of one-way and two-way coupling methods for numerical analysis of fluid-structure interactions. Journal of Applied Mathematics.

Chen, W. F. (2007). Plasticity in reinforced concrete. J. Ross Publishing.

Degroote, J., Haelterman, R., Annerel, S., Bruggeman, P., and Vierendeels, J. (2010). Performance of partitioned procedures in fluidstructure interaction. Computers \& Structures, 88(78):446 - 457.

Dettmer, W. and Peri, D. (2008). On the coupling between fluid flow and mesh motion in the modelling of fluidstructure interaction. Computational Mechanics, 43(1):81-90.

Donea, J. and Huerta, A. (2003). Finite element methods for flow problems. Wiley, England.

Habchi, C., Russeil, S., Bougeard, D., Harion, J. L., Lemenand, T., Ghanem, A., Valle, D. D., and Peerhossaini, H. (2013). Partitioned solver for strongly coupled fluidstructure interaction. Computers \& Fluids, 71(0):306 -319 .

Jo, C. H., Kim, D. Y., Rho, Y. H., Lee, K. H., and Johnstone, C. (2013). Fsi analysis of deformation along offshore pile structure for tidal current power. Renewable Energy, 54(0):248 - 252.

Kalkan, E. and Sevilgen, V. (2011). March 11, 2011 m9.0 tohoku, japan earthquake: Preliminary results. United States Geological Survey. 
Lim, W. Z., Xiao, R., and Chin, C. S. (2012). A comparison of fluid-structure interaction methods for a simple numerical analysis of concrete gravitydam. Proceedings of the 20th UK Conference of the Association for Computational Mechanics in Engineering .

Lim, W. Z., Xiao, R., T., H., and Chin, C. S. (2013). Partitioned methods in computational modelling on fluid-structure interactions of concrete gravity-dam. Computer and Information Science.

Ma, S. and Mahfuz, H. (2012). Finite element simulation of composite ship structures with fluid structure interaction. Ocean Engineering, 52(0):52 59 .

Michler, C., Hulshoff, S., van Brummelen, E., and de Borst, R. (2004). A monolithic approach to fluidstructure interaction. Computers \& Fluids, $33(56): 839-848$.

Richter, T. (2010). Numerical methods for fluid-structure interaction problems. University of Heidelberg.

Song, M., Lefranois, E., and Rachik, M. (2013). A partitioned coupling scheme extended to structures interacting with high-density fluid flows. Computers \& Fluids, 84(0):190 - 202.

Stansberg, C., Baarholm, R., Kristiansen, T., Hansen, E., and Rortveit, G. (2005). Extreme wave amplication and impact loads on offshore structures. Offshore Technology Conference.

Versteeg, H. and Malalasekera, W. (2007). An introduction to computational 285 fluid dynamics the finite volume method. Pearson Education Limited, England.

Walhorn, E., Klke, A., Hbner, B., and Dinkler, D. (2005). Fluid structure coupling within a monolithic model involving free surface flows. Computers \& Structures, 83(2526):2100 - 2111.

290 Wall, W. A., Genkinger, S., and Ramm, E. (2007). A strong coupling partitioned approach for fluidstructure interaction with free surfaces. Computers \& Fluids, 36(1):169 - 183. 
Zhang, J., Guo, L., Wu, H., Zhou, A., Hu, D., and Ren, J. (2014). The influence of wind shear on vibration of geometrically nonlinear wind turbine blade under fluidstructure interaction. Ocean Engineering, 84(0):14 - 19. 